\title{
Synthesis of Bis (n-salicylidene-n-phenyl aminato) Palladium (II) complex to prepare a number of biphenyl compounds
}

\author{
Md. A. Hashem*, S. K. Tuli and T. B. Mahmud
}

Department of Chemistry, Jahangirnagar University, Savar, Dhaka-1342, Bangladesh

\begin{abstract}
The non-toxic, stable, Schiff base complex of $\mathrm{Na}_{2} \mathrm{PdCl}_{4}$ and $\mathrm{N}$-salicylidene-N-phenyl amine in methanol catalyze the Suzuki-Miyaura coupling reaction to synthesize coupling products (Scheme-1) in good to excellent yields with high purity under mild conditions.

Received: 12 August 2018

Revised: 03 October 2018

Accepted: 03 December 2018

DOI: $10.3329 /$ bjsir.v54i3.42674

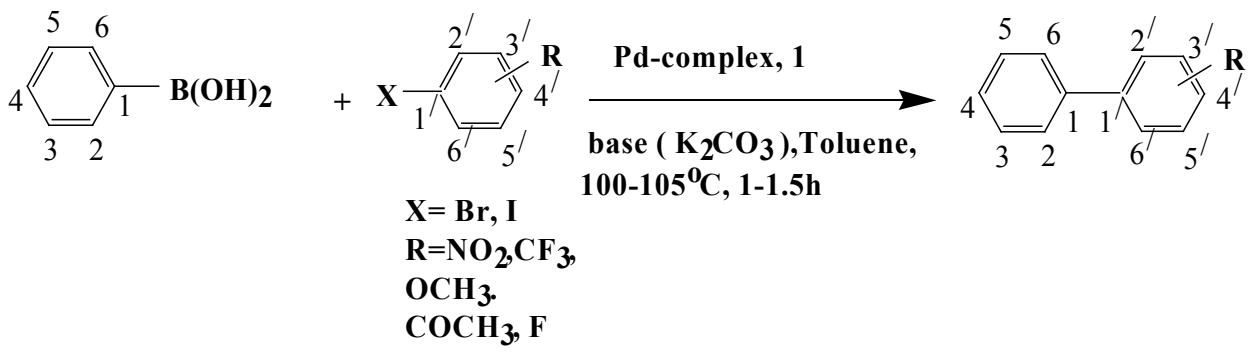

Scheme-1

Keywords: Catalysis; Non-toxic palladium complex; Coupling reaction; Phenyl boronic acid

\section{Introduction}

Organopalladium chemistry is one of the most important areas of organometallic chemistry directed towards organic synthesis. Several features which make reactions involving Pd catalysts and reagents particularly useful and versatile among many transition metals used for organic synthesis (Dupont et al.,1997; Girlingn, 1982). Most importantly, Pd catalysts offer an abundance of possibilities of carbon-carbon, carbon-nitrogen bond formation. Organopalladium compounds are among the most readily available and easily prepared and handled of the plethora of known transition metal complexes.

Recently Schiff base Pd complexes (Singleton, 2003) have found wide applications in the organic synthesis. So, we have synthesized a new palladium (II) Schiff base complex and tested its catalytic activity in Suzuki-Miyaura cross coupling reactions.

\section{Materials and methods}

Preparation of the ligand, $N$-salicylidene- $N$-phenyl amine

2- Hydroxybenzaldehyde, (10 $\mathrm{mmol} \approx 1.22 \mathrm{~g})$ was dissolved in $15 \mathrm{~mL}$ ethanol (absolute). Four drops of conc. $\mathrm{HCl}$ acid were added to it. Then with stirringethanolic solution of aniline $(10.3 \mathrm{mmol} \cong 0.96 \mathrm{~g}$ in $10 \mathrm{~mL}$ ethanol) was added. The reaction mixture was refluxed for three hours until completion of the reaction (TLC checkup); the reaction mixture was kept for one hour under ice- cooling. It was neutralized with saturated solution of $\mathrm{NaHCO}_{3}$. On adding ice into the flask it was kept in a refrigerator for overnight. A yellow solid was precipitated out and was collected after filtration and kept in a vaccumdesicator for drying. After recrystalization from EtOHa shiny yellow needle - shaped crystal, mp.41 ${ }^{\circ} \mathrm{C},(173 \mathrm{mg}, 91 \%)$ was obtained.
\end{abstract}


Synthesis of $P d$ complex catalyst, 1 by complexation of the ligand with $\mathrm{Na}_{2} \mathrm{PdCl}_{4}$

At first the ligand $(1 \mathrm{mmol} \approx 197 \mathrm{mg}$ ) was dissolved in $4 \mathrm{~mL}$ of methanol (absolute). Then with stirring sodiumtetrachloropalladate, $\mathrm{Na}_{2} \mathrm{PdCl}_{4}(442 \mathrm{mg} \approx 1.50 \mathrm{mmol})$ was added, immediate precipitation with coloration of the either activated molecules or harsh reaction conditions. The mild reaction condition for coupling reactions using the Pd-catalyst, 1 was developed in our laboratory to form C-C bonding in a one step process.

Application of the prepared catalyst, 1

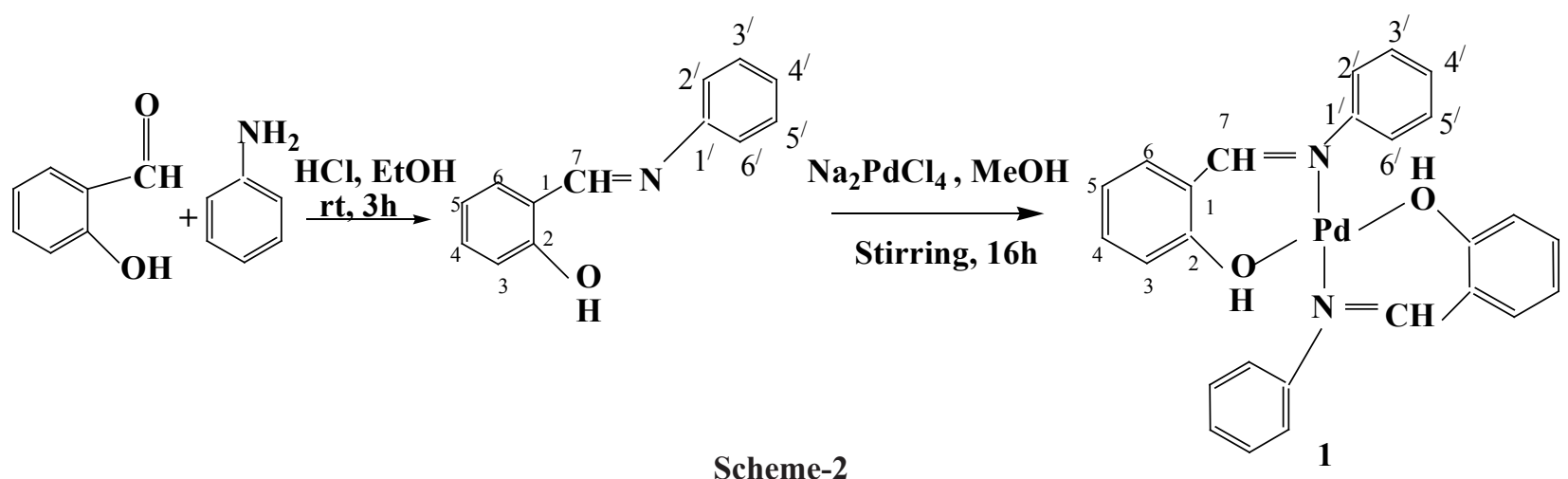

mixture was observed. The stirring was continued for another 16 hours (overnight) at room temperature. The reaction mixture was filtered and washed with $5 \mathrm{~mL}$ of $\mathrm{MeOH}$ and the yellow solid was collected. The material was dried in high vacuum until constant weight. The yield of the solid complex, 1 was $242 \mathrm{mg}$ (97\%) and the melting point was 250 ${ }^{\circ} \mathrm{C}$ (decomposition).

General procedure for Suzuki-Miyaura cross coupling reaction utilizing the complex, 1

Phenyl boronic acid (1.5 $\mathrm{mmol})$, aryl halide (1 $\mathrm{mmol})$, catalyst, $1(1 \mathrm{mmol} \%)$, base $\left(\mathrm{K}_{2} \mathrm{CO}_{3}, 2 \mathrm{mmol}\right)$ and toluene $(3.5 \mathrm{~mL})$ were mixed together. The mixture was refluxed at $105^{\circ} \mathrm{C}$ for $1-2$ hours. The mixture was cooled to room temperature, diluted with $\mathrm{H}_{2} \mathrm{O}(10 \mathrm{~mL})$ and extracted three times withethyl acetate $(10 \mathrm{~mL})$; any residual solids present were filtered. The organic phase was dried over anhydrous sodium sulphate and evaporated in rotary evaporator. The crude product was column chromatographed over a silica gel eluting with $\mathrm{PE}$ : $\mathrm{DCM}=(10: 1)$ solvent mixture to obtain pure products.

\section{Results and discussion}

The palladium catalyzed reactions are extremely powerful tools for the formation of C-C, C-N, C-O, C-S,C-P and C-Se bonds. The mild reaction conditions of these reactions offer considerable advantages over classical methods that require
The activity of the Pd-complex, 1 as catalyst was tested in a number of Suzuki-MiyauraCross Coupling reactions between aryl halides and aryl boronic acids. The reaction of aryl boronic acids with substituted aryl halides using the complex 1 gave different derivatives of substituted biphenyls as coupling products (Table I). From reaction no 1-11 phenyl boronic acid was used \& in no-12 para-chlorophenyl boronic acid was used.

Reaction condition: Phenyl boronic acid (1.5 mmol), aryl halide (1mmol), base $\left(\mathrm{K}_{2} \mathrm{CO}_{3}, 2 \mathrm{mmol}\right)$ and the Pd-complex $1(1 \mathrm{mmol} \%)$ intoluene $(3.5 \mathrm{~mL})$ refluxed at $105^{\circ} \mathrm{C}$ for $1-2$ hours.

On the basis of the reaction time and yield it was observed that the aryl halides substituted with electron withdrawing groups had higher yield than the aryl halides substituted with electron donating groups. Due to crowding effect aryl halides substituted at ortho-position were less reactive to the coupling reaction than the meta- and para-derivatives.

The reaction of bromo derivatives need more time and the yield of the bromo derivatives were higher than the iodo derivatives. Aryl boronic acids substituted with electron withdrawing group (no. 12) was less reactive than the phenyl boronic acid.Important biological compounds such as 1, 1'-Biphenyl, 4-(Trifluormethyl)biphenyl, 4-Acetyl biphenyl, 1, 3-Diphenylbenzene, 2-phenylanisol with high purity were synthesized in a one step process. 
Table I. The SUZUKI-MIYAURA cross coupling reaction utilizing the Pd complex 1\&aryl boronic acid

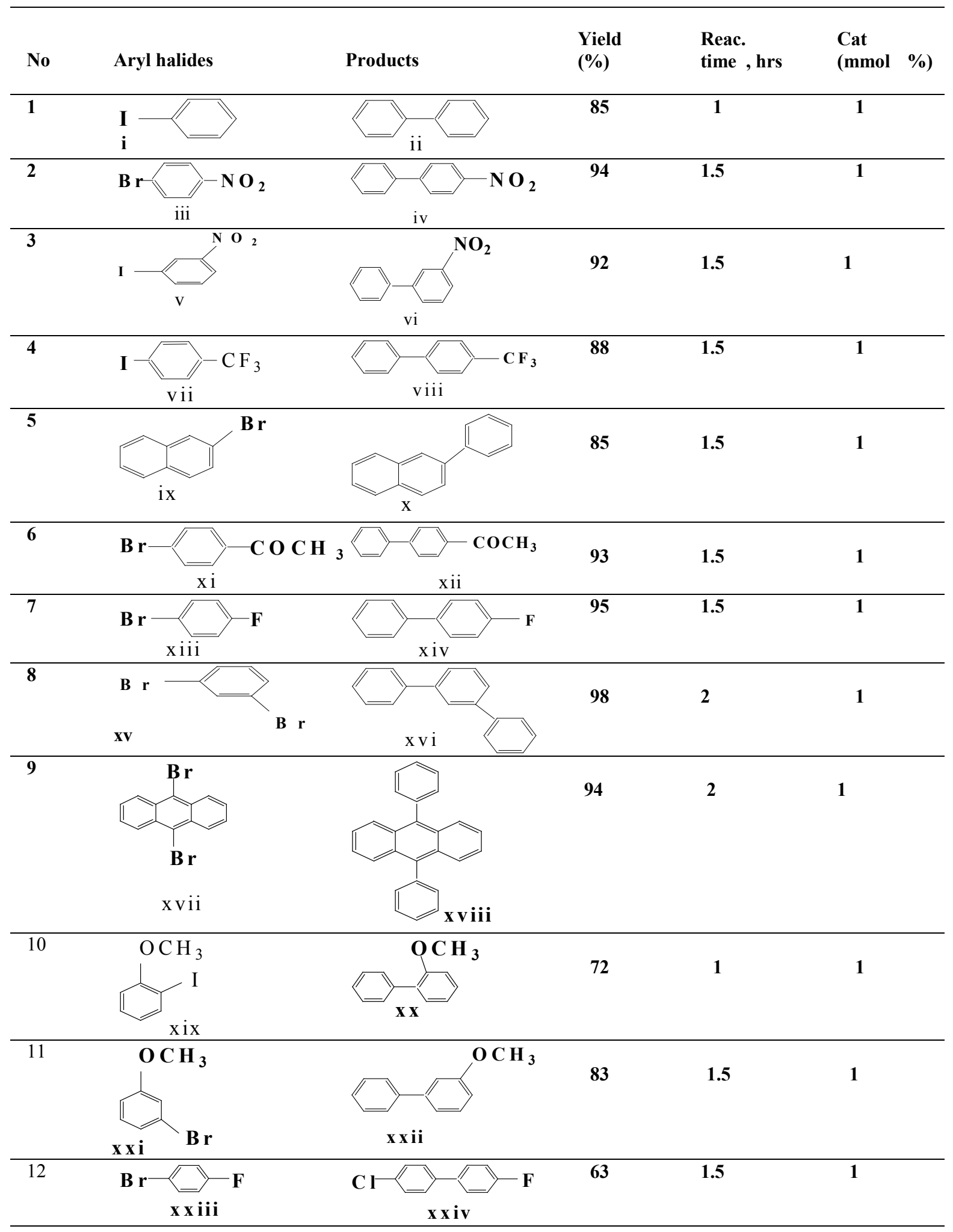


Analytical and spectral data of the compounds

$N$-salicylidene- $N$-phenyl amine

$1.73 \mathrm{mg}(91 \%), \mathrm{mp} .41^{\circ} \mathrm{C}, \mathrm{FT}-\mathrm{IR} \quad(v): 3448 \quad(\mathrm{OH}, \quad$ broad $)$, 3054(aro. C-H str.), $1616(\mathrm{C}=\mathrm{N}$, str. $), 1589,1570(\mathrm{C}=\mathrm{C})$, 1357, 1275(C-N, str.), 1185(C-O, str.) $\mathrm{cm}^{-1} .{ }^{1} \mathrm{H}-\mathrm{NMR}(400$ $\left.\mathrm{MHz}, \mathrm{CDCl}_{3}\right) \delta(\mathrm{ppm}): 13.26(1 \mathrm{H}, \mathrm{s}, \mathrm{OH}), 8.61(1 \mathrm{H}, \mathrm{s}, \mathrm{H}-7)$, 7.40(4H,m, H-2',3',5',6'), 7.28 (3H, m, H- 4,5,6 ), 7.04(1H, d, H-3), 6.95(1H, t, H-4'). ${ }^{13} \mathrm{C}$ - NMR: 162(C-7), 161(C-2), 148(C-1), 148(C-1'), 129(C-3', 5'), 121(C-2', 4'). Mass Spectra: $197\left[\mathrm{M}^{+}\right], 196[\mathrm{M}-1]^{+}$.

\section{Bis (N-salicylidene-N-phenyl aminato) palladium} (II) complex, 1

$242 \mathrm{mg}(97 \%), \mathrm{mp} . \quad 250^{\circ} \mathrm{C}($ decoposition). FT-IR ( $v): 3380$ $(\mathrm{OH}), 3286$ (alip. $=\mathrm{C}-\mathrm{H}), 3204,3120$ (aro. $=\mathrm{C}-\mathrm{H}$, str.), 1606 $(\mathrm{C}=\mathrm{N}), 1573,1493,1464$ (aro. $\mathrm{C}=\mathrm{C}), 1319,1259$ (C-N, single str.), 1115 (C-O, str.).cm ${ }^{-1} .{ }^{1} \mathrm{H}-\mathrm{NMR}\left(400 \mathrm{MHz}, \mathrm{CDCl}_{3}\right.$ ) $\delta(\mathrm{ppm}): 10.01(\mathrm{H}-8, \mathrm{~s}, 1 \mathrm{H}), 7.68(\mathrm{H}-6, \mathrm{dd}, 1 \mathrm{H}, \mathrm{J}=8 \mathrm{~Hz}, 2 \mathrm{~Hz})$, 7.48-7.55(H-4', m, 1H), 7.32 (H-2',6', dd, 2H, J=7.6 Hz, $2.00 \mathrm{~Hz}), 7.15$ (H-3',5', dt, 2H ), 6.81 (H-4, 5, dt,2H, J=8Hz, $0.08 \mathrm{~Hz}), 6.77(\mathrm{H}-3$,dd, $1 \mathrm{H}, \mathrm{J}=8 \mathrm{~Hz}, 1 \mathrm{~Hz}), 5.59(\mathrm{H}-7, \mathrm{~s}$, 1H).Mass Spectra: $499\left([\mathrm{M}+\mathrm{H}]^{+}, 100 \%\right), 498\left(\mathrm{M}^{+}, 98 \%\right)$, $497\left([\mathrm{M}-\mathrm{H}]^{+}, 65 \%\right), 500\left([\mathrm{M}+2 \mathrm{H}]^{+}, 60 \%\right)$.

1,1'-Biphenyl, ii:131mg (85\%), mp.68-70 ${ }^{\circ} \mathrm{C} .{ }^{1} \mathrm{H}-\mathrm{NMR}(400$ $\left.\mathrm{MHz}, \mathrm{CDCl}_{3}\right) \quad \delta(\mathrm{ppm}): 7.62\left(4 \mathrm{H}, \mathrm{dd}_{,} \mathrm{J}_{0}=7.2 \mathrm{~Hz}, \mathrm{~J}_{\mathrm{m}}=1.6 \mathrm{~Hz}\right.$, H-2,2',6,6'), 7.46(4H,dd, J $\left.=7.6 \mathrm{~Hz}, 7.2 \mathrm{~Hz}, \mathrm{H}-3,3^{\prime}, 5,5^{\prime}\right), 7.36(2 \mathrm{H}$ $\left., \mathrm{dt}, \mathrm{J}_{0}=7.6 \mathrm{~Hz}, \mathrm{~J}_{\mathrm{m}}=1.6 \mathrm{~Hz}, \mathrm{H}-4,4^{\prime}\right) \cdot{ }^{13} \mathrm{C}-\mathrm{NMR} \delta(\mathrm{ppm}): 141.20$ $\left(\mathrm{C}-1,1^{\prime}\right), \quad 128.72$ (C-2,2',6,6'), 127.22 (C-4,4'), 127.14 $\left(\mathrm{C}-3,3^{\prime}, 5,5^{\prime}\right)$ DEPT $135 \quad-128.72 \quad\left(\mathrm{C}-2,2^{\prime}, 6,6^{\prime}\right)$, $127.22\left(\mathrm{C}-4,4^{\prime}\right), 127.14\left(\mathrm{C}-3,3^{\prime}, 5,5^{\prime}\right)$. M a s s Spectra:ESI $(+\mathrm{ve}): \mathrm{m} / \mathrm{z}: 331[2 \mathrm{M}+\mathrm{Na}-\mathrm{H}]^{+}, 193.2[\mathrm{M}+\mathrm{K}]^{+}$, ESI(-ve):m/z:307[2M-H] $]^{-}, 217.2[\mathrm{M}+\mathrm{OAc}]^{-}, 189.4\left[\mathrm{M}+{ }^{35} \mathrm{Cl}\right]^{-}$

4-Nitrobiphenyl,iv:187mg (94\%), mp.114-116 ${ }^{\circ} \mathrm{C} .{ }^{1} \mathrm{H} \quad-$ $\mathrm{NMR}\left(400 \quad \mathrm{MHz}, \quad \mathrm{CDCl}_{3}\right) \delta(\mathrm{ppm}): 8.29\left(2 \mathrm{H}, \mathrm{d}, \mathrm{J}_{0}=8.8 \mathrm{~Hz}\right.$, $\mathrm{H}-2,6), 7.73\left(2 \mathrm{H}, \mathrm{d}, \mathrm{J}_{0}=8.8 \mathrm{~Hz}, \mathrm{H}-3,5\right), 7.62\left(2 \mathrm{H}, \mathrm{dd}, \mathrm{J}_{0}=7.2 \mathrm{~Hz}, \mathrm{~J}_{\mathrm{m}}=\right.$ 1.6Hz,H-2',6'), 7.49(2H,dd, $\left.\mathrm{J}_{0}=7.2 \mathrm{~Hz}, 7.6 \mathrm{~Hz}, \mathrm{H}-3^{\prime}, 5^{\prime}\right), 7.45(1 \mathrm{H}$, $\left.\mathrm{dt}, \mathrm{J}_{0}=7.6 \mathrm{~Hz}, \mathrm{~J}_{\mathrm{m}}=1.6 \mathrm{~Hz}, \mathrm{H}-4{ }^{\prime}\right) . \quad{ }^{13} \mathrm{C} \quad-\mathrm{NMR} \delta(\mathrm{ppm})$ : 147.59(C-1), 147.02(C-4), $138.72 \quad$ (C-1'), 129.12 $(\mathrm{C}-2,6), 128.89$ (C-3,5),127.76(C-2',6'), 127.37(C-3',5'), 124.07(C-4').DEPT 135-129.12(C-2,6),128.89 (C-3,5), 127.76(C-2',6'), 127.37(C-3',5'),124.07(C-4'). Mass Spectra: ESI(+ve):m/z: $222.2 \quad[\mathrm{M}+\mathrm{Na}]^{+}, \mathrm{ESI} \quad(-\mathrm{ve}): \quad \mathrm{m} / \mathrm{z}: 258.3$ $\left[\mathrm{M}+\mathrm{OAc}^{-}, 199[\mathrm{M}]^{+}\right.$

3-Nitrobiphenyl, vi:183mg (92\%),mp.59-60 ${ }^{\circ} \mathrm{C} .{ }^{1} \mathrm{H}$ - NMR $\left(400 \mathrm{MHz}, \mathrm{CDCl}_{3}\right) \delta(\mathrm{ppm}): 8.45\left(1 \mathrm{H}, \mathrm{dd}_{,} \mathrm{J}_{\mathrm{m}}=1.6 \mathrm{~Hz}, 2 \mathrm{~Hz}\right.$,
$\mathrm{H}-2), 8.19\left(1 \mathrm{H}, \mathrm{dd}, \mathrm{J}_{0}=8.0 \mathrm{~Hz}, \mathrm{~J}_{\mathrm{m}}=1.2 \mathrm{~Hz}, \mathrm{H}-6\right), 7.90(1 \mathrm{H}, \mathrm{dd}$, $\left.\mathrm{J}_{0}=7.6 \mathrm{~Hz}, \mathrm{~J}_{\mathrm{m}}=1.2 \mathrm{~Hz}, \mathrm{H}-4\right), 7.58-7.63\left(3 \mathrm{H}, \mathrm{m}, \mathrm{H}-5 \quad \& \mathrm{H}-2^{\prime}, 6^{\prime}\right)$, $7.49\left(2 \mathrm{H}, \mathrm{t}, \mathrm{J}_{0}=7.2 \mathrm{~Hz}, \mathrm{H}-3^{\prime}, 5^{\prime}\right), 7.43\left(1 \mathrm{H}, \mathrm{dt}, \mathrm{J}_{0}=7.2 \mathrm{~Hz}, \mathrm{~J}_{\mathrm{m}}=1.2 \mathrm{~Hz}\right.$, $\left.\mathrm{H}-4^{\prime}\right) .{ }^{13} \mathrm{C}-\mathrm{NMR} \delta$ (ppm):148(C-1), $142(\mathrm{C}-3), 138\left(\mathrm{C}-1^{\prime}\right)$, 133(C-2), 129.67(C-6), 129.12(C-4), 128.50(C-5), 127.77(C-2' ,6'),121.99(C-3',5'),121.91(C-4').DEPT 135 ${ }^{\circ}-133(\mathrm{C}-2)$, 129.67 (C-6),129.12(C-4),128.50(C-5),127.77(C-2',6'), 121.99 (C-3',5'),121.91(C-4').Mass Spectra: ESI (+ve): m/z: $222.2[\mathrm{M}+\mathrm{Na}]^{+}$, ESI (-ve): m/z: $258.3[\mathrm{M}+\mathrm{OAc}]^{-}$.

4-(Trifluormethyl)biphenyl, viii: 195mg (88\%), mp.70-72 ${ }^{\circ}$ C.FT-IR $\left(\mathrm{cm}^{-1}\right)$ : 3072(aro. C-H str.), 1614 and 1490 (aro. $\mathrm{C}=\mathrm{C}$ str.),843(para sub. bending of aro. C-H), 668(C-F str.). ${ }^{1} \mathrm{H}-\mathrm{NMR}\left(300 \mathrm{MHz}, \mathrm{CDC}_{3}\right) \delta(\mathrm{ppm}) 7.68(4 \mathrm{H}, \mathrm{s}(\mathrm{br}), \mathrm{H}-$ $2,3,5,6), 7.58\left(2 \mathrm{H}, \mathrm{dt}, \mathrm{J}_{0}=6.9 \mathrm{~Hz}, \mathrm{~J}_{\mathrm{m}}=1.8 \mathrm{~Hz}, \mathrm{H}-2^{\prime}, 6^{\prime}\right), 7.46(2 \mathrm{H}, \mathrm{dt}, \mathrm{J}$ $\left.=6.9 \mathrm{~Hz}, \mathrm{~J}_{\mathrm{m}}=1.8 \mathrm{~Hz}, \mathrm{H}-3^{\prime}, 5^{\prime}\right), 7.39\left(1 \mathrm{H}, \mathrm{dt}, \mathrm{J}_{0}=7.2 \mathrm{~Hz}, \mathrm{~J}_{\mathrm{m}}=1.8 \mathrm{~Hz}, \mathrm{H}\right.$ $\left.{ }^{-4}\right) . \quad{ }^{13} \mathrm{C} \quad$ - NMR $\delta(\mathrm{ppm}): 144.70(\mathrm{C}-4), 139.75(\mathrm{C}-1)$, 129.71(C-1'), 129.53(C-3,5),128.97(C-2,6), 127.40(C-2',6'), $127.26\left(\mathrm{C}-3^{\prime}, 5^{\prime}\right), 126.11$ ( C - 7) 125.77 ( C - $\left.4^{\prime}\right)$. DEPT-135-129.53(C-3,5), 128.97(C-2,6), 127.40(C-2',6'), 127.26(C-3',5'),126.11(C-7), 125.77(C-4'). Mass Spectra: $\mathrm{ES} \mathrm{I}(+\mathrm{ve}): \mathrm{m} / \mathrm{z}: 242.3[\mathrm{M}+\mathrm{N} \text { a }]^{+}$, ESI(-ve):m/z:253.5[M+ $\left.+{ }^{35} \mathrm{Cl}\right]^{-}, 255.5\left[\mathrm{M}+{ }^{37} \mathrm{Cl}\right]^{-}$

2-phenylnapthalene, $\quad \mathrm{x}: \quad 204 \mathrm{mg} \quad(85 \%), \quad \mathrm{mp} .105^{\circ} \mathrm{C} .{ }^{1} \mathrm{H}-$ $\mathrm{NMR}\left(400 \mathrm{MHz}, \mathrm{CDCl}_{3}\right) \delta(\mathrm{ppm}): 8.05(1 \mathrm{H}, \mathrm{s}, \mathrm{H}-1), 7.92(1 \mathrm{H}, \mathrm{d}$, $\left.\mathrm{J}_{0}=8.4 \mathrm{~Hz}, \mathrm{H}-3\right), 7.90\left(1 \mathrm{H}, \mathrm{d}, \mathrm{J}_{0}=8.4 \mathrm{~Hz}, \mathrm{H}-4\right), 7.89\left(1 \mathrm{H}, \mathrm{dd}, \mathrm{J}_{0}=6.8\right.$ $\mathrm{Hz}, 7.2 \mathrm{~Hz}, \mathrm{H}-8), 7.88(1 \mathrm{H}, \mathrm{d}, \mathrm{J}=6.8 \mathrm{~Hz}, \mathrm{H}-7), 7.71-7.77(3 \mathrm{H}, \mathrm{m}, \mathrm{H}-$ $5,6,7), 7.46-7.54\left(4 \mathrm{H}, \mathrm{H}-2^{\prime}, 3^{\prime}, 5^{\prime}, 6^{\prime}\right), 7.39\left(1 \mathrm{H}, \mathrm{dt}, \mathrm{J}_{0}=6.8 \mathrm{~Hz}, 7.2 \mathrm{~Hz}\right.$ ,H-4'). ${ }^{13} \mathrm{C} \quad-\quad \mathrm{NMR} \delta \quad(\mathrm{ppm}): 141.1(\mathrm{C}-2), 138.53\left(\mathrm{C}-1^{\prime}\right)$, 133.64(C-9),132.58(C-10),128.84,128.39,128.17,127.62,12 $7.41,127.33,126.27,125.91,125.78,125.58$. DEPT-135-128.84, 128.39, 128.17, 127.62, 127.41, 127.33, 126.27, 125.91, 125.78, 125.58.Mass Spectra: ESI (+ve): $\mathrm{m} / \mathrm{z}: 247[\mathrm{M}+42+\mathrm{H}]^{+}, \mathrm{ESI}(-\mathrm{ve}): \mathrm{m} / \mathrm{z}: 206[\mathrm{M}-\mathrm{H}]^{-}$

4-Acetylbiphenyl, xii:182mg(93\%),mp. $\quad 117-118^{\circ} \mathrm{C} .{ }^{1} \mathrm{H} \quad-$ $\mathrm{NMR}\left(400 \mathrm{MHz}, \mathrm{CDCl}_{3}\right) \delta(\mathrm{ppm}): 8.02\left(2 \mathrm{H}, \mathrm{d}, \mathrm{J}_{0}=8.4 \mathrm{~Hz}, \mathrm{H}-2,6\right)$, $7.68\left(2 \mathrm{H}, \mathrm{d}, \mathrm{J}_{0}=8.4 \mathrm{~Hz}, \mathrm{H}-3,5\right), 7.63\left(2 \mathrm{H}, \mathrm{dd}, \mathrm{J}_{0}=7.2 \mathrm{~Hz}, \mathrm{~J}_{\mathrm{m}}=1.2 \mathrm{~Hz}\right.$, H-2', 6'), 7.46(2H,dt, $\left.\mathrm{J}_{0}=7.2 \mathrm{~Hz}, \mathrm{~J}_{\mathrm{m}}=1.2 \mathrm{~Hz}, \mathrm{H}-3{ }^{\prime}, 5^{\prime}\right), 7.39(1 \mathrm{H}, \mathrm{dt}, \mathrm{J}$ $\left.=7.2 \mathrm{~Hz}, \mathrm{~J}_{\mathrm{m}}=1.2 \mathrm{~Hz}, \mathrm{H}-4{ }^{\prime}\right), 2.63\left(3 \mathrm{H}, \mathrm{s}, \mathrm{CH}_{3}\right) \cdot{ }^{13} \mathrm{C}-\mathrm{NMR} \delta$ (ppm): $\quad$ 197.74(C-7, $\quad \mathrm{C}=\mathrm{O}), 145.74 \quad(\mathrm{C}-1), 139.8(\mathrm{C}-4)$, 135.8(C-1'), 128.93(C-3,5), 128.66(C-2,6), 128.20 (C-2',6'), 127.24(C-4'), $127.19\left(\mathrm{C}-3^{\prime}, 5^{\prime}\right), 26.65\left(\mathrm{C}-8, \mathrm{CH}_{3}\right)$. DEPT- $135^{\circ}-$ 128.93(C-3,5), 128.66(C-2,6),128.20 (C-2',6'), 127.24(C-4'), 127.19(C-3',5'),26.65(C-8, $\left.\mathrm{CH}_{3}\right)$. Mass Spectra: ESI (+ve): $\mathrm{m} / \mathrm{z}: 414[2 \mathrm{M}+\mathrm{Na}-\mathrm{H}]^{+}, \quad 219.1[\mathrm{M}+\mathrm{Na}]^{+}, \quad$ ESI $(-\mathrm{ve}): \mathrm{m} / \mathrm{z}$ : 255.3[M+OAc]

4-Fluorobiphenyl, xiv:164mg (95\%), mp. $74-76^{\circ} \mathrm{C} .{ }^{1} \mathrm{H}-$ NMR $\quad\left(300 \quad \mathrm{MHz}, \quad \mathrm{CDCl}_{3}\right) \delta(\mathrm{ppm}): 7.50-7.62$ 
( $4 \mathrm{H}, \mathrm{m}, \mathrm{H}-2,2,, 6,61)$, $7.43\left(2 \mathrm{H}, \mathrm{dd}, \mathrm{J}_{0}=7.2 \mathrm{~Hz}, 7.5 \mathrm{~Hz}, \mathrm{H}-3^{\prime}, 5^{\prime}\right), 7.33\left(1 \mathrm{H}, \mathrm{dt}, \mathrm{J}_{0}=\right.$ $\left.7.5 \mathrm{~Hz}, \mathrm{H}-4^{\prime}\right), 7.12\left(2 \mathrm{H}, \mathrm{dt}, \mathrm{J}_{0}=8.7 \mathrm{~Hz}, \mathrm{~J}_{\mathrm{m}}=2.1 \mathrm{~Hz}, \mathrm{H}-3,5\right) \cdot{ }^{13} \mathrm{C} \quad-$ NMR $\delta \quad(p p m): \quad 164(\mathrm{C}-1), 160.80(\mathrm{C}-4), 141.22\left(\mathrm{C}-1^{\prime}\right)$, 138.97(C-2,6),137.32(C-3,5),137.28(C-2',6'), 128.79(C-3',5') ,115.72(C-2,6). DEPT-135- 128.97(C-2, 6), 137.32(C-3, 5), 137.28(C-2', 6'), 128.79(C-3', 5'),115.72(C-2,6). Mass Spectra: ESI $(+\mathrm{ve}): \mathrm{m} / \mathrm{z}: 193[\mathrm{M}+\mathrm{Na}]^{+}$, ESI $(-\mathrm{ve}): \mathrm{m} / \mathrm{z}$ : $228.4[\mathrm{M}+\mathrm{OAc}-\mathrm{H}]$

1, 3-Diphenylbenzene, xvi: 226mg (98\%),86-88 ${ }^{\circ} \mathrm{C} .{ }^{1} \mathrm{H}-$ NMR (400 MHz, $\left.\mathrm{CDCl}_{3}\right) \delta(\mathrm{ppm}): 7.81\left(1 \mathrm{H}, \mathrm{t}, \mathrm{J}_{\mathrm{m}}=1.8 \mathrm{~Hz}, \mathrm{H}-2\right)$, $7.65\left(4 \mathrm{H}, \mathrm{dd}, \mathrm{J}_{0}=6.9 \mathrm{~Hz}, \mathrm{~J}_{\mathrm{m}}=2.1 \mathrm{~Hz}, \mathrm{H}-2^{\prime}, 2^{\prime \prime}, 6^{\prime}, 6^{\prime \prime}\right), 7.59(2 \mathrm{H}, \mathrm{m}, \mathrm{H}-$ 4,6), 7.52(1H,dd,J=6.0Hz,5.4Hz,H-5), 7.48(4H,m,H-3',3",5',5 "), $7.37\left(2 \mathrm{H}, \mathrm{dt}, \mathrm{J}_{0}=7.1 \mathrm{~Hz}, \mathrm{~J}_{\mathrm{m}}=2.1 \mathrm{~Hz}, \mathrm{H}-4\right.$ ',4")

9,10-diphenylantracene, xviii: $317 \mathrm{mg}(94 \%), 251-254^{\circ} \mathrm{C} . F T-I R$ $\left(\mathrm{cm}^{-1}\right)$ : 3065(aro.C-H str.), 1598 and 1481(aro.C $=\mathrm{C}$ str.) 2-phenylanisol, $\quad \mathrm{xx}: 133 \mathrm{mg} \quad(72 \%), \quad$ bp. $>250^{\circ} \mathrm{C} . \quad$ FT-IR $\left(\mathrm{cm}^{-1}\right): 3072 \quad$ (aro.C-H str.),2922(assy.alp.C-H str.), 2854(sym.alp.C-H str.), 1602 and 1485(aro. C=C str.), 1461 and 1347 ( $\mathrm{CH}_{3}$ ben.), 842 (para sub. bending of aro. $\mathrm{C}-\mathrm{H}), 765$ and 691 (mono sub. bending of aro. C-H).

3-phenylanisol, $\quad$ xxii: $\quad 151.7 \mathrm{mg} \quad(83 \%), \quad \mathrm{mp} . \quad 250^{\circ} \mathrm{C}$. FT-IR( $\mathrm{cm}^{-1}$ ): 3034(aro.C-H str.),2941(assy.alp.C-H str.), 2838 (sym.alp.C-H str.),1599 and1479(aro. C=C str.), $1296\left(\mathrm{CH}_{3}\right.$ ben.), $\quad 1179(\mathrm{C}-\mathrm{O} \quad$ str.) Mass Spectra: ESI $(\mathrm{m} / \mathrm{z}): 184.5[\mathrm{M}]^{+}, 183\left[\mathrm{M}^{+}-\mathrm{H}\right], 182\left[\mathrm{M}^{+}-\mathrm{H}_{2}\right], 169.5\left[\mathrm{M}^{+}-\mathrm{CH}_{3}\right], 153$ $.8\left[\mathrm{M}^{+}-\mathrm{OCH}_{3}\right]$

4-Floro-4-Chloro-biphenyl, xxiv: 132.1mg (63\%), mp-90-93 ${ }^{\circ} \mathrm{C}$. FT-IR $\left(\mathrm{cm}^{-1}\right): 3051$ (aro.C-H str.), 1601 and 1480 (aro.C $=\mathrm{C}$

str.),1157(aro.C-F str.),1090(aro.C-Cl str.). Mass Spectra: ESI (m/z):208.5:206.5(3:1) [M] $]^{+}, 187.5: 189.5(3: 1) \quad\left[\mathrm{M}^{+}-\mathrm{F}\right]$, $171\left[\mathrm{M}^{+}-\mathrm{Cl}\right], 170\left[\mathrm{M}^{+}-\mathrm{HCl}\right], 152\left[\mathrm{M}^{+}-\mathrm{Cl}-\mathrm{F}\right]$.

Reaction mechanism: The mechanism for the Suzuki coupling reaction of aryl boronic acids with aryl halides can be represented according to the literature (Casado,1998)as shown below:

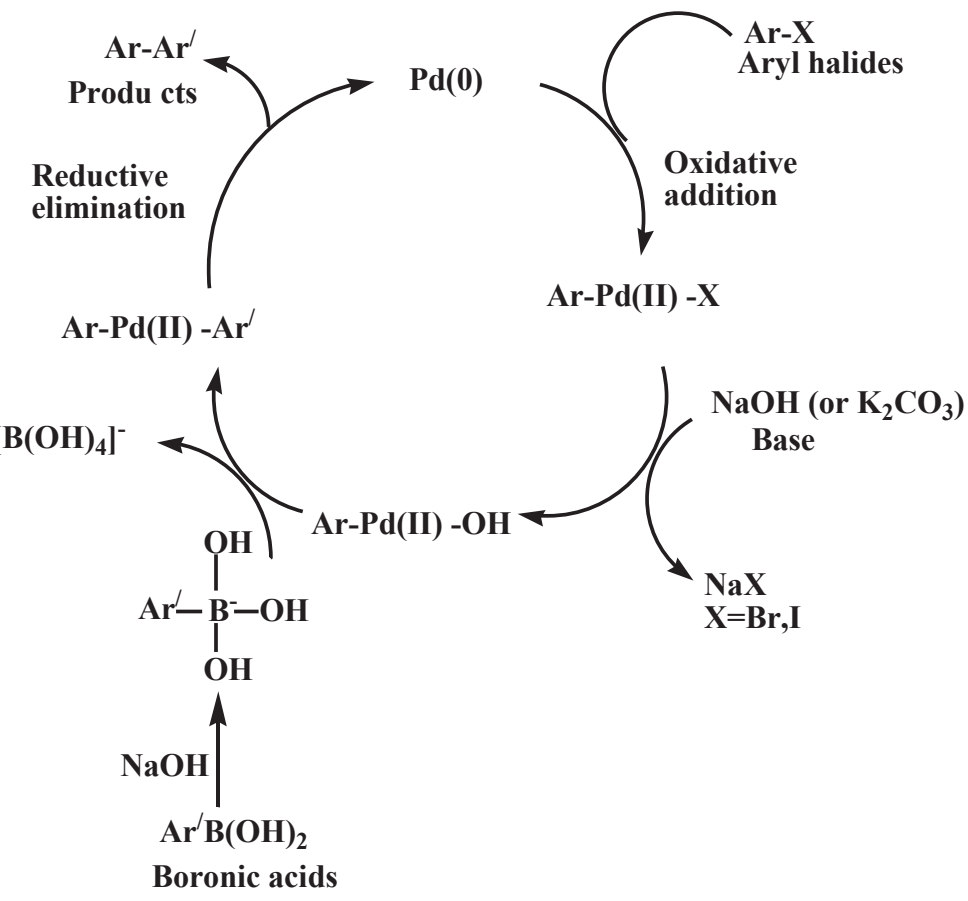

Fig. 1. Mechanism for the Suzuki coupling reaction using Palladiumcomplex, 1 
Spectra of the ligand \& the catalyst
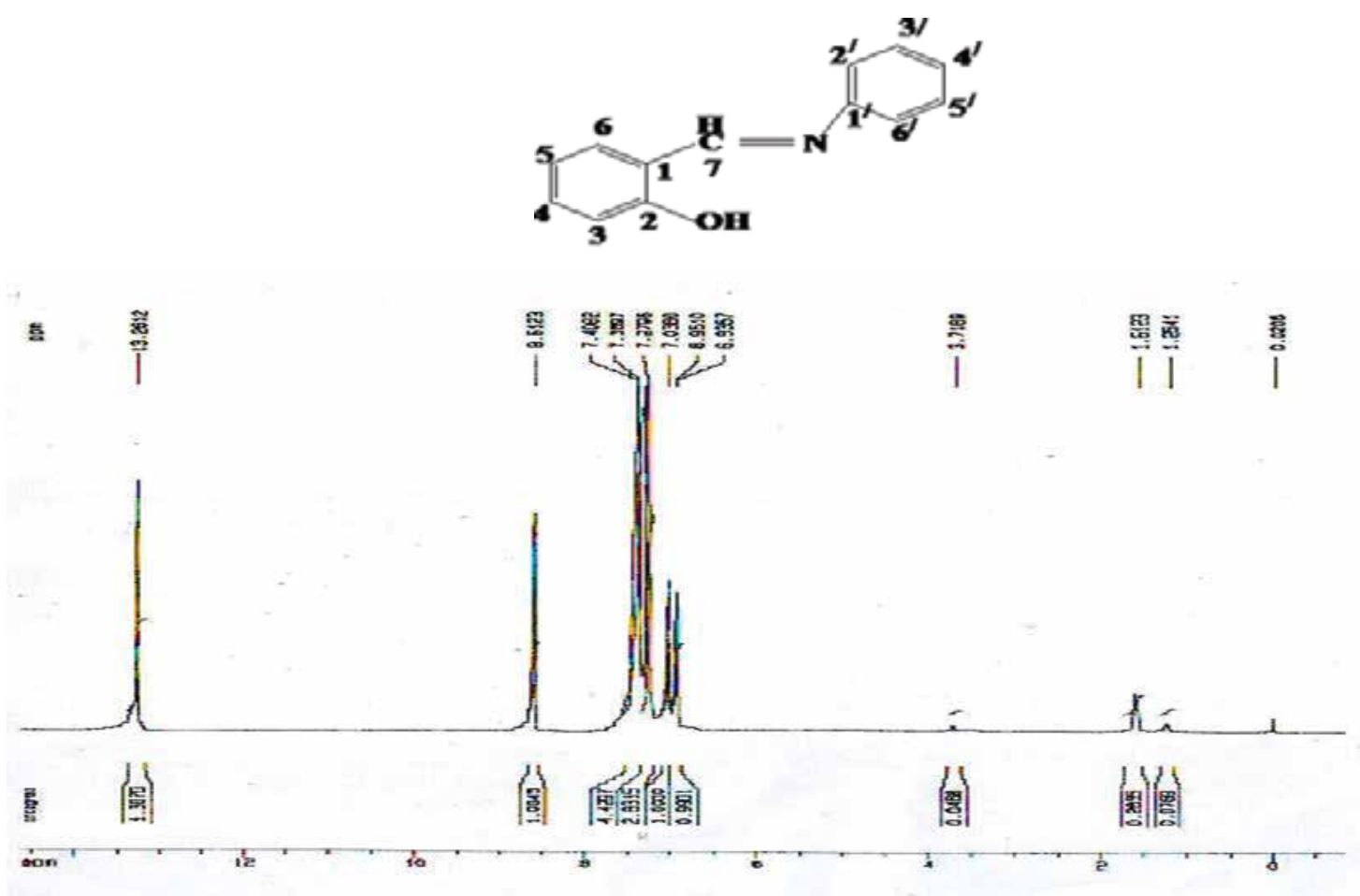

Fig. 2. 1H-NMR of the ligand



Fig. 3. Mass spectra of the ligand 


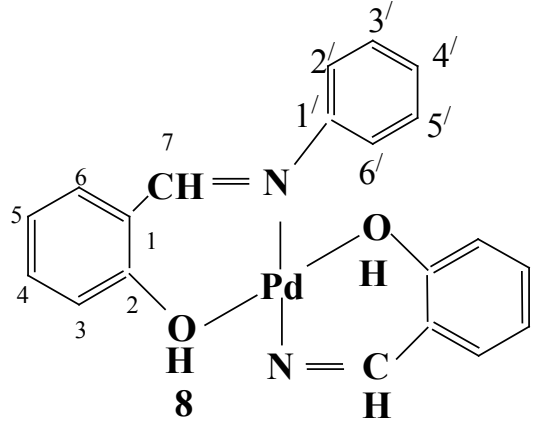

Palladium catalyst

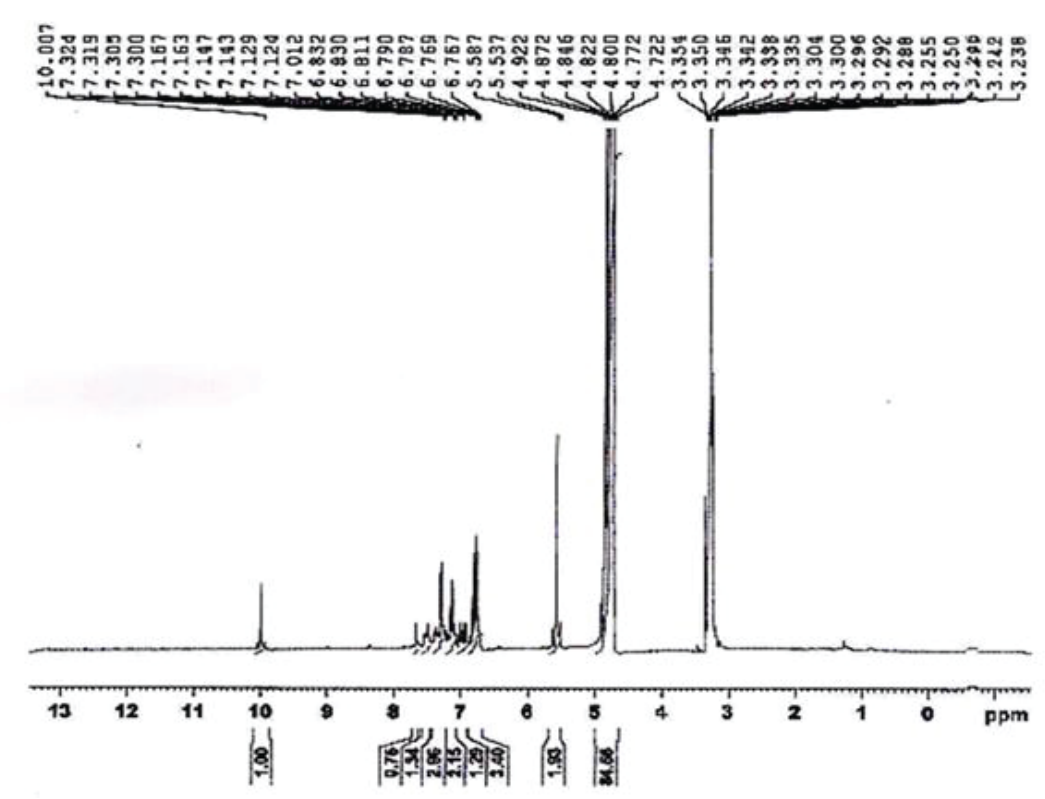

Fig. 4. ${ }^{1} \mathrm{H}-\mathrm{NMR}$ of the Pd-catalyst

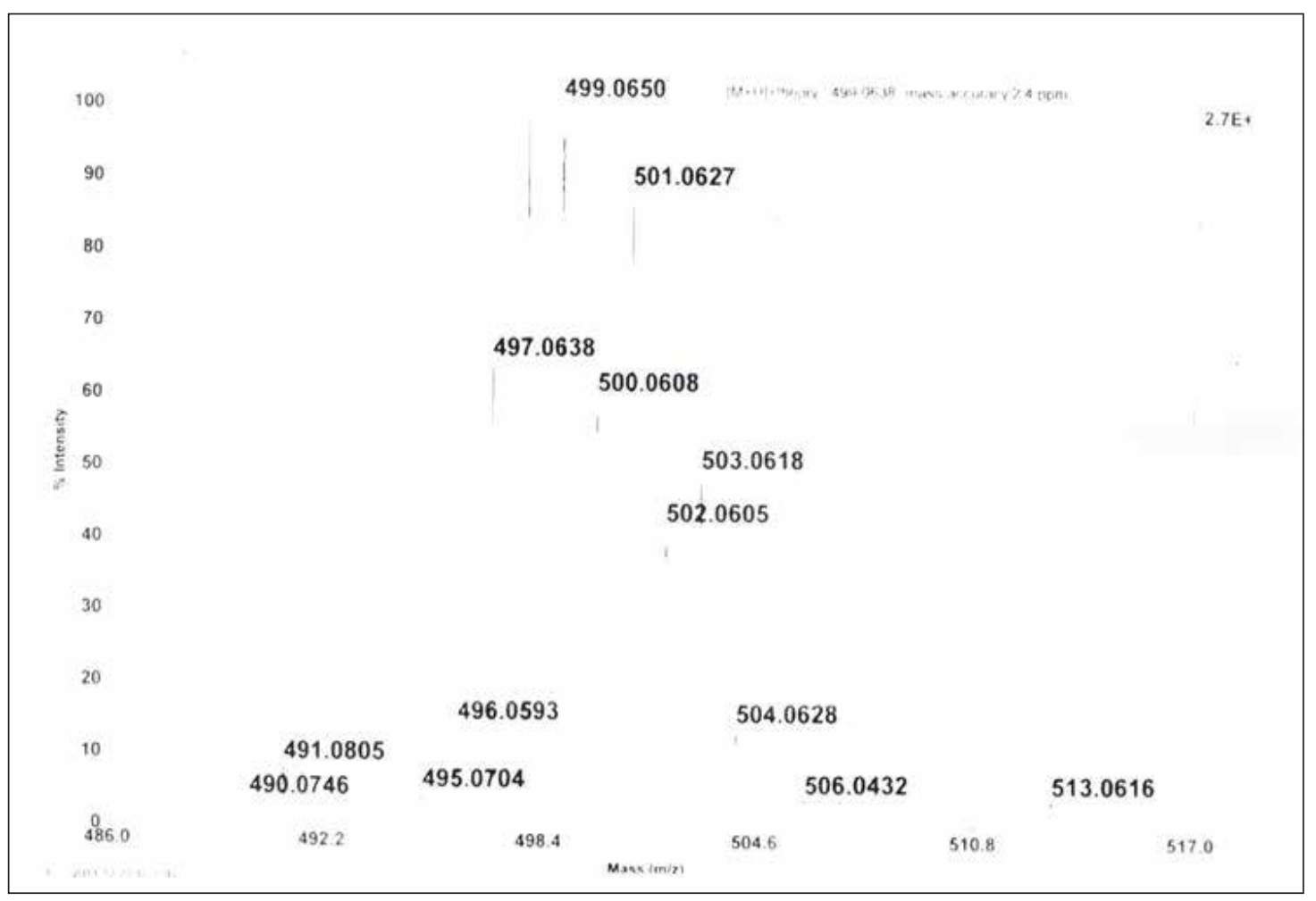

Fig. 5. Mass spectra of the Pd-catalyst 


\section{Conclusion}

In summary, an efficient, environment friendly, inexpensive and new Palladium (II) complex 1 has been successfully used as catalyst in a number of Suzuki-Miyaura cross coupling reactions between aryl halides and aryl boronic acidsunder mild conditions. From synthetic point of view, a new, effective \& single step method has been developed to form $\mathrm{C}-\mathrm{C}$ bond in good to excellent yields with high purity.

\section{Acknowledgement}

The authors express their thanks to Jahangirnagar University \&Leibniz Institute of Plant Biochemistry, Halle (Saale), Germany and BCSIR for recording spectra.

\section{References}

Casado AL, Espinet P and Gallego A M (1998), On the Configuration Resulting from Oxidative Addition of $\mathrm{RX}$ to $\mathrm{Pd}$ (PPh3) 4 and the Mechanism of the cis-to-trans Isomerization of [PdRX(PPh3)2] Complexes $(\mathrm{R}=$ Aryl, $\mathrm{X}=$ Halide $)$, Organometallics 17: $954-959$.

Dupont J and Basso N (1997), The trans-Chloropalladation Reaction of Propargyl Amines and Thioethers, Organometallics 16: 2386-2391.
Girlingn I Rand and Widdowson DA (1982), Cyclopalladated imines in synthesis 2 : a new synthesis of isoquinolines, Tetrahedron Lett. 23:1957. DOI: org/10. 1021/om970064r

Matos K and Soderquist J A (1998) Alkylboranes in the Suzuki-Miyaura Coupling: Stereochemical and Mechanistic Studies, J. Org. Chem. 63: 461-470. DOI: org/10.1021/jo971681s

Stille JK (1977), Mechanisms of oxidative addition of organic halides to Group 8 transition-metal complexes, Acc. Chem. Res. 10: 434-442.

Singleton, JT (2003), The Uses of Pincer Complexes in Organic Synthesis, Tetrahedron 59: 1837-1857

Vicente J, Saura-Llamas I and Jones PG (1993), Orthometallated primary amines. Part 1. Facile preparation of the first optically active cyclopalladated primary amines, J. Chem. Soc. Dalton Transactions 23: $3619-3624$. 